\title{
Acceptability and feasibility of end-of-life care pathways in Australian residential aged care facilities
}

\section{Dell E Horey BAppSc, MMedSc(ClinEpi), PhD Research Fellow \\ Annette F Street BEd(Hons), PhD Professor of Palliative Care}

Alison F Sands MB BS(Hons), FRACGP General Practitioner

1 La Trobe University, Melbourne, VIC.

2 North East Valley Division of General Practice Melbourne, VIC

d.horey@latrobe.edu.au

MJA 2012; 197: 106-109 doi: 10.5694/mjall.11518 $\square$ nd-of-life (EOL) care pathways are specialised integrated care pathways for the terminal phase of palliative care. Traditionally associated with cancer care, EOL care pathways aim to support quality palliative care by improving symptom management, documentation and assessment. ${ }^{1,2}$ Evidence of effectiveness from randomised controlled trials is not yet available, ${ }^{2}$ although other study types from several countries consistently support the use of EOL care pathways. ${ }^{3-6}$ They are increasingly used internationally and are appearing in Australian residential aged care facilities (RACFs) ${ }^{7,8}$ as active documents that provide guidance on different aspects of terminal care. ${ }^{9}$

Effectiveness is just one requirement of evidence-based practice. Acceptability by those receiving and providing care and feasibility in the particular clinical and social context are also critical. ${ }^{10}$ These criteria have particular importance in complex care environments such as RACFs where care is provided by a mix of nursing and allied health staff, personal care assistants and general practitioners. ${ }^{11}$ EOL care pathways support multidisciplinary care teams to document decisions, actions and observations ${ }^{12}$ and may also be useful quality markers of care at the end of life. ${ }^{13}$

EOL care pathways were introduced to 14 RACFs in Victoria and South Australia through A good death in residential aged care: optimising the use of medicines to manage symptoms in the end-of-life phase (the Good Death project). The Good Death project used a modified version of the widely used Liverpool Care Pathway for the Dying Patient, ${ }^{14}$ adapted and evaluated for use in Australian RACFs. ${ }^{15}$ We aimed to examine the acceptability and feasibility of using EOL care pathways in RACFs in Australia.

\section{Methods}

The Good Death project involved a consortium of partners across Victoria

Objectives: To investigate the acceptability and feasibility of using end-of-life (EOL) care pathways in residential aged care facilities (RACFs).

Design, setting and participants: Multistage action research approach involving interviews, surveys and prospective audits of deaths and EOL care pathway use among residents and staff of RACFs and associated general practitioners from 14 RACFs in Victoria and South Australia between April 2009 and July 2010.

Intervention: Introduction of EOL care pathways.

Main outcome measures: Evidence of acceptability was determined by the rate of pathway use in RACFs and through feedback from RACF managers, staff and GPs. Evidence of feasibility was determined by reductions in transfers to hospital for symptom management before death, length of time on pathways, and whether care was consistent with best practice at EOL.

Results: The use of EOL care pathways across the RACFs fell into low-, moderate- and high-uptake groups (for 10\%, 34\% and $68 \%$ of all deaths at the facility, respectively). Feedback from RACF staff and GPs indicated that acceptability was critical to successful implementation. The use of EOL care pathways demonstrated improvements in care, sometimes over extended periods. There were fewer unnecessary admissions to hospital before death, although not all RACF staff and GPs were aware of the project.

Conclusion: EOL care pathways are feasible strategies for delivering EOL care consistent with best practice. However, their introduction into Australian RACFs needs to include strategies to facilitate acceptability by RACF staff and GPs.

and South Australia led by the North East Valley Division of General Practice, Victoria. It included two other Divisions of General Practice, 14 RACFs with a total of 1033 resident places, pharmacists, specialist services, and research staff from La Trobe University, Melbourne. There was considerable heterogeneity across the RACFs (Box 1).

The project used a multistage action research design that collected both qualitative and quantitative data from a range of sources. Data were obtained each month from the 14 participating RACFs by project staff using purpose-designed tools. Data sources included interviews with RACF staff and GPs, RACF manager surveys, and the prospective audits of deaths and EOL care pathway use. RACF staff reviewed retrospective records of residents over the last 28 days of life to examine if common signs of transition to death were evident. All RACF managers ${ }^{14}$ were surveyed at the start of the project in April 2009 and again in July 2010. Baseline and post-implementation interviews were conducted with 28 GPs (21 at baseline and seven post-implementation) and 42 RACF staff members (17 registered nurses, seven enrolled nurses, 14 personal care workers and four allied health workers).

EOL care pathways were introduced to the RACFs from June 2009 and prospective audits of all deaths in participating RACFs were undertaken from 1 November 2009 to 31 July 2010. The audit sought details about each death, including information about hospital transfers, use of EOL care pathways, actions recorded in pathways, and the initiators and signatories of pathway actions.

The 12-month reports from RACF managers before and after implementation provided two comparable periods to assess hospital transfers and were used to determine feasibility. The death audits confirmed that use of the EOL care pathways was consistent with best-practice care and were used to determine acceptability.

The EOL care pathway used in the Good Death project had five main sections: (i) commencing a pathway; (ii) medical interventions and advance care planning; (iii) care staff interven- 
tions, including care management, daily comfort care chart and further care action sheet; (iv) multidisciplinary communication sheet; and (v) after-death care. The care team, including the GP, decided whether it was appropriate to commence a resident on the pathway or to reconsider its continuance. ${ }^{16}$ The pathway could be initiated by a GP or a registered nurse and local protocols were developed. As only non-identifying data were collected, patient consent was not required. Our study was approved by the La Trobe University Human Ethics Committee.

\section{Measures}

Acceptability is associated with preferences and values. Evidence of the acceptability of EOL care pathways was determined a priori to be demonstrated by the rate of pathway use and through feedback from RACF managers, staff and GPs. We sought views regarding changes to the approach to people who were dying, including perceptions of the involvement of residents and families in decision making, staff confidence in managing care, and collaboration between staff and GPs.

Feasibility is associated with practical implementation. Evidence of the feasibility of using EOL care pathways was determined a priori to be demonstrated by reductions in transfers to hospital for symptom management before death, length of time on pathways, and whether care was consistent with best practice at EOL as determined by national guidelines. $^{17,18}$

\section{Data analysis}

Consistent with an action research approach, we generated data to meet evaluation objectives and undertook quantitative and qualitative analyses.

All quantitative data were coded as either dichotomous or categorical variables and entered into PASW Statistics, version 17 (SPSS Inc, Chicago, Ill, USA). We calculated simple descriptive statistics and set significance at 0.05 for all analyses. Mantel-Haenszel $\chi^{2}$ summary statistics were calculated for reported death data from all RACFs. This procedure adjusts for confounding from different mortality rates to give a measure of the average strength of association between variables.

\begin{tabular}{|c|c|c|}
\hline Area of difference & Type of difference & No. of patients \\
\hline \multirow[t]{3}{*}{ Level of care } & High care only & 2 \\
\hline & Low care only & 1 \\
\hline & Mix of high and low care (including ageing in place) & 11 \\
\hline Size & 30-134 places per RACF & \\
\hline \multirow[t]{2}{*}{ Location } & Urban & 12 \\
\hline & Outer metropolitan/rural & 2 \\
\hline \multirow[t]{3}{*}{ Cultural specificity } & Ethnospecific (Greek, Italian) & 2 \\
\hline & Monocultural & 4 \\
\hline & Mix of ethnic backgrounds & 8 \\
\hline \multirow[t]{2}{*}{ Profit status } & For profit & 4 \\
\hline & Not for profit & 10 \\
\hline \multirow[t]{3}{*}{ RACF provider } & Single, privately owned & 6 \\
\hline & Part of multichain & 7 \\
\hline & Colocated with public hospital & 1 \\
\hline Staff profile & \multicolumn{2}{|c|}{ Staff-to-resident, nurse-to-carer, full-time-to-part-time-staff ratios } \\
\hline General practitioner involvement & \multicolumn{2}{|c|}{ 3-39 per RACF; mean patients per GP, 5.7 (range, 1-65) } \\
\hline \multicolumn{3}{|l|}{ Use of Medication Imprest system ${ }^{\dagger}$} \\
\hline South Australia & \multicolumn{2}{|l|}{5 out of 5 RACFs } \\
\hline Victorian & \multicolumn{2}{|l|}{2 out of 9 RACFs } \\
\hline \multicolumn{3}{|c|}{ Access to specialist palliative care and other services } \\
\hline Victoria & \multicolumn{2}{|c|}{$\begin{array}{l}\text { Community palliative care services provide clinical support and education } \\
\text { to RACFs, including specialist nurses to work with local RACFs and GPs. } \\
\text { Hospital outreach provides care to RACF residents and support staff to } \\
\text { prevent unnecessary transfers to emergency departments }\end{array}$} \\
\hline South Australia & \multicolumn{2}{|c|}{$\begin{array}{l}\text { Extended Care Paramedic Program (SA Health and the SA Ambulance } \\
\text { Service) manages and treats people in their usual residence }\end{array}$} \\
\hline
\end{tabular}

We collected the major sources of qualitative data by telephone interviews, and transcribed and imported the data into NVivo, version 8 (QSR International, Melbourne, Vic). These data were subject to qualitative content analysis, which identified core consistencies to generate a comprehensive analysis informed by the project objectives. $^{19}$

\section{Results}

\section{Acceptability}

Over the 9-month death audit period, of a total 1033 RACF resident places, 175 (17\%) residents died; equivalent to an annual death rate of $23 \%$. Pathways were used 63 times $(36 \%$ of all deaths and $43 \%$ of deaths when sudden deaths not on pathways were excluded). Pathways were considered for another four residents not on a pathway when they died. Three other residents received care from community palliative care services and were not on a pathway.

There were three levels of uptake of EOL care pathways across the 14
RACFs. A high-uptake group (four RACFs) used pathways for $68 \%$ of all deaths $(93 \%$ of deaths when sudden deaths not on pathways were excluded); a moderate-uptake group (six RACFs) used pathways for $34 \%$ of all deaths (41\% when sudden deaths not on pathways were excluded); and a low- or no-uptake group (four RACFs) used pathways for $10 \%$ of all deaths (11\% when sudden deaths not on pathways were excluded). The difference between the groups was highly significant $(P<0.0001)$ and remained when sudden deaths not on pathways were excluded. Heterogeneity across the RACFs was also apparent across the groups, and uptake was not associated with any known factors.

Registered nurses initiated most EOL care pathways (57/63, 90\%). GPs initiated five of 63 pathways $(8 \%)$ and were signatories to about half the pathways $(31,49 \%)$ in 10 of the 14 RACFs. RACF staff reported verbal support from some GPs unable to attend the RACF in person. The initiator of one pathway was not recorded. 
2 Number and place of resident deaths and transfers to hospital for the previous 12 months, reported by residential aged care facility (RACF) managers at baseline and post-implementation*

Baseline $\left(\%^{\dagger}\right)$ Post-implementation $\left(\%^{\dagger}\right)$ Mantel-Haenszel $\chi^{2}$

\begin{tabular}{lcc}
\hline Total deaths in previous 12 months & $267\left(26 \%{ }^{\ddagger}\right)$ & $274\left(27 \%^{\ddagger}\right)$ \\
Deaths in RACF & $229(86 \%)$ & $232(85 \%)$ \\
Deaths in hospital & $38(14 \%)$ & $42(15 \%)$ \\
Transfers to hospital & $52(19 \%)$ & $44(16 \%)$ \\
Transferred to hospital and died on return to RACF & $14(5 \%)$ & $2(1 \%)$ \\
\hline * Baseline, April 2008 to April 2009; post-implementation, June 2009 to June 2010. † Proportion of total deaths in previous \\
12 months, unless otherwise specified. $¥$ Of 1033 beds. $\$ P=0.002$.
\end{tabular}

3 Impact of pathway on end-of-life care, by rate of uptake

Pathway*

Medicines ordered as needed

Inappropriate interventions and observations discontinued
Non-essential medicines discontinued

High $(n=25)$ Moderate $(n=34)$ Low $(n=4)$ All $(n=63)$

$\begin{array}{llll}25 & 33 & 4 & 62(98 \%) \\ 19 & 26 & 3 & 48(76 \%) \\ 16 & 19 & 3 & 38(60 \%)\end{array}$

*As determined by best-practice guidelines. ${ }^{17,18}$

Feedback from RACF managers in the high-uptake group indicated pathway use was incorporated into policy and had become routine care, and two RACFs linked pathway use to their auditing processes. One manager reported staff "enthusiasm to start pathways". No managers from moderate-uptake RACFs described policy changes related to the pathway. Low uptake of pathways was attributed to either few deaths in the audit period, so little opportunity to use them, or the lack of an electronic version.

GP involvement appeared influential to the level of uptake. Highuptake RACFs were more likely to have pathways signed by a GP than moderate-uptake RACFs (20/24 [83\%] v 9/32 [28\%]; $P<0.001)$.

In the initial interviews with 21 GPs, nine were satisfied with how EOL care was managed at the RACFs they attended, although their views tended to be qualified or concerned the competence of particular staff. Three GPs raised concerns about variability in EOL care and the timeliness of their involvement.

During the project, GPs in 10 of the 14 RACFs were directly involved in implementing EOL care pathways. In addition, five of the seven GPs interviewed at the end of the project commented positively about their experience. The other two GPs were unaware of the project or pathways.

One GP felt the project provided a catalyst for discussion about death among doctors, nursing staff, and family and relatives. He also noted the RACFs' expectation that GPs manage the care of dying residents more scientifically, using their pharmacological and medical skills. Another GP commented on other beneficial effects, such as easier dose management and more streamlined care.

Two GPs with wide palliative care experience noted positive effects among RACF staff and an improved understanding of the dying process. One reflected that staff were more aware of the principles and plans involved, which increased staff confidence in how they cared for patients and also enabled them to voice any concerns. He observed that the pathway is part of an education process, "where most people go from trying to salvage people no matter what, to a process whereby people are allowed to die peacefully".

\section{Feasibility}

Indications of important practice change were evident in RACF managers' reports of transfers to hospital in the period before death. The proportion of deaths in hospital and RACFs remained constant in pre- and postimplementation manager surveys, but significantly fewer residents were transferred to hospital and subsequently returned to the RACF at the end of the project (Box 2).

The audit confirmed the reported mortality rates and place of death by the managers, including the important reduction in unnecessary hospital transfers. The proportion of deaths in hospital remained comparable to baseline data, but only one of the 18 people in the audit group transferred to hospital returned to the RACF. One resident on a pathway was transferred to hospital for pain management and subsequently died there.

The median length of time on an EOL care pathway for residents was 5.5 days (SD, 25.3). RACF managers reported that before the introduction of pathways, their reviews of resident records revealed that little was written about the care provided. The pathways encouraged documentation, and the audits demonstrated that care for residents on pathways was consistent with best practice at EOL, regardless of an RACF's level of pathway uptake. Box 3 shows how aspects of care promoted by the project were implemented. Almost all people on a pathway had appropriate medicines ordered as needed. Nonessential medicines were discontinued for $76 \%$ of those on pathways, and inappropriate interventions and observations were discontinued for $60 \%$ of those on pathways.

Approach to care was raised in most post-implementation interviews with RACF staff (25/42, 60\%). One in four reported no change. However, most reported improvements, such as more holistic or improved quality of care $(13,31 \%)$; increased focus on EOL and palliative care $(19,45 \%)$; more consistent care $(15,36 \%)$; more timely care (11, $26 \%)$; more systematic care (11, $26 \%)$; and more informed decision making $(3,7 \%)$.

Greater consistency in the approach to care was evident in several comments. One nurse commented that it is a "a more holistic approach, not just looking at the person and their needs, but going through all the needs". A manager observed that previously, "it all relied on [an] individual nurse's knowledge to make an assessment, and put things in place, and now there is a ... more structured approach".

The use of EOL care pathways demonstrated improvements in care, sometimes over extended periods, and fewer unnecessary admissions to hospital before death were noted, although not all RACF staff and GPs were aware of the project. 


\section{Discussion}

When introducing EOL care pathways in Australian RACFs, acceptability and feasibility are important considerations to ensure that quality EOL care is sustained in practice. Our measures for acceptability and feasibility are consistent with studies of EOL care pathways in acute settings, which found positive responses from nurses associated with increased confidence and better relationships with medical staff; ${ }^{20}$ audits of EOL care pathway use showed improved EOL care. $^{3}$

We used the rate of uptake of EOL care pathways as part of our measure of acceptability, recognising that pathways are not suitable for every death. The significant differences in uptake across the 14 RACFs were associated with acceptability of pathways by RACF staff and GPs. This indicates that acceptability is crucial to successful implementation of EOL care pathways.

We found a similar level of uptake to that found among six Queensland RACFs. ${ }^{8}$ Reymond and colleagues attributed higher uptake to greater support from management and GPs based on anecdotal accounts from RACF staff, consistent with our notion of acceptability. The Queensland study also reported that retention of a common link nurse throughout the project to be an important factor for uptake. As ongoing sustainability was our goal, we did not introduce additional staff into the RACFs. We found higher uptake to be related to reported change in RACF policies.

Our study provided evidence of feasibility in using EOL care pathways in RACFs consistent with best practice at EOL. There was appropriate anticipatory planning of medicines and discontinuation of nonessential medications, interventions and observations when pathways were used across all RACFs, regardless of level of uptake.
Our study limitations include the bias of all observational studies. Participating RACFs were self-selected and clustering was likely. Experiences in using an EOL care pathway may affect subsequent decisions about its use, although this would apply both for and against uptake. There is no consensus on the best measures for acceptability and feasibility of pathway use in RACFs, but we selected a mix of quantitative and qualitative parameters to enable our assessment and chose these a priori to minimise bias.

EOL care pathways are feasible mechanisms for delivering EOL care consistent with best practice. Strategies to facilitate acceptability by RACF staff and GPs include incorporating EOL care pathways into existing standards and practices, and promoting awareness, education and accessibility. Acceptability of EOL care pathways could be achieved by establishing a national program to support their use (including support for training, implementation and monitoring); developing an electronic version; obtaining endorsement from the Royal Australian College of General Practitioners; emphasising their use in RACF accreditation; and incorporating them into advance care planning discussions.

Acknowledgements: We thank the residential aged care facilities, their residents and staff involved in this project. We also thank Mary Belfrage, Diana Cooper, Melanie Rayner and Clare Chiminello (North East Valley Division of General Practice), South Australian project officers (Adelaide North East Division of General Practice and General Practice Network South), and Angela Herd and Bronwyn Carter (La Trobe University). The Good Death project was funded by the Australian Government Department of Health and Ageing under the Encouraging Best Practice in Residential Aged Care program.

Competing interests: No relevant disclosures.

Received 28 Nov 2011, accepted 23 Apr 2012.

1 Chan R, Webster J. End-of-life care pathways for improving outcomes in caring for the dying. Cochrane Database Syst Rev 2010; (1): CD008006.

2 Costantini M, Ottonelli S, Canavacci L, et al; LCP Randomised Italian Cluster Trial Study Group. The effectiveness of the Liverpool care pathway in improving end of life care for dying cancer patients in hospital. A cluster randomised trial. BMC Health Serv Res 2011; 11: 13.
3 Le BH, Watt JN. Care of the dying in Australia's busiest hospital: benefits of palliative care consultation and methods to enhance access. JPalliat Med 2010; 13: 855-860.

4 Hockley J, Watson J, Oxenham D, Murray SA. The integrated implementation of two end-of-life care tools in nursing care homes in the UK: an indepth evaluation. Palliat Med 2010; 24: 828-838.

5 Bailey FA, Burgio KL, Woodby LL, et al. Improving processes of hospital care during the last hours of life. Arch Intern Med 2005; 165: 1722-1727.

6 Veerbeek L, van Zuylen L, Swart SJ, et al. The effect of the Liverpool Care Pathway for the dying: a multi-centre study. Palliat Med 2008; 22 145-151.

7 Seymour JE, Kumar A, Froggatt K. Do nursing homes for older people have the support they need to provide end-of-life care? A mixed methods enquiry in England. Palliat Med 2011; 25: 125-138.

8 Reymond L, Israel FJ, Charles MA. A residential aged care end-of-life care pathway (RAC EoLCP) for Australian aged care facilities. Aust Health Rev 2011; 35: 350-356.

9 Centre for Palliative Care Research and Education. End of life care pathways. Brisbane: Queensland Government, 2012. http:// www.health.qld.gov.au/cpcre/eol_pthwys.asp (accessed Mar 2012).

10 Haynes RB, Devereaux PJ, Guyatt GH. Physicians' and patients' choices in evidence based practice. BMJ 2002; 324: 1350 .

11 O'Halloran J, Britt H, Valenti L. General practitioner consultations at residential agedcare facilities. Med J Aust 2007; 187: 88-91.

12 Ellershaw J, Dewar S, Murphy D. Achieving a good death for all. BMJ 2010; 341: 44861 .

13 Pugh EJ, McEvoy M, Blenkinsopp J. Use of the proportion of patients dying on an End of Life pathway as a quality marker: considerations for interpretation. Palliat Med 2010; 24: 544-547.

14 Marie Curie Palliative Curie Institute. Liverpool Care Pathway for the dying patient (LCP). Liverpool: National Health Service, 1999. http:// www.liv.ac.uk/mcpcil/liverpool-care-pathway/ (accessed Apr 2011).

15 Brisbane South Palliative Care Collaborative. Residential Aged Care End-of-Life Care Pathway (RAC EoLCP) Project: summary report. Brisbane: BSPCC, 2009.

16 Evans $G$. Improving end of life care for the person with dementia: a practical approach from general practice. Dementia 2009; 8: 363-376. doi: 10.1177/1471301209104978.

17 National Health and Medical Research Council. Guidelines for a palliative approach in residential aged care. Enhanced version - May 2006. Canberra: Commonwealth of Australia, 2006.

18 Palliative Care Expert Group. Therapeutic guidelines: palliative care. Version 3. Melbourne: Therapeutic Guidelines Limited, 2010.

19 Patton MQ. Qualitative research and evaluation methods. 3rd ed. Thousand Oaks, CA: Sage, 2002.

20 O'Hara T. Nurses' views on using the Liverpool care pathway in an acute hospital setting Int J Palliat Nurs 2011; 17: 239-244. 